\title{
X-ray Characterization of Films Formed by Pulsed Laser Deposition on Cold Substrates from YBaCuO Targets
}

\author{
J.B. PeŁka ${ }^{a}$, W. Paszkowicz ${ }^{a}$, P. GierŁowski ${ }^{a}$, \\ S.J. LeWANDOWSKI ${ }^{a}$, M. Zieliński ${ }^{a}$, S. BARBANERA ${ }^{b}$ \\ AND M. KNAPP ${ }^{c}$ \\ ${ }^{a}$ Institute of Physics, Polish Academy of Sciences \\ al. Lotników 32/46, 02-668 Warsaw, Poland \\ ${ }^{b}$ Institute of Solid State Electronics CNR \\ via Cineto Romano 42, 00-156 Rome, Italy \\ ${ }^{c}$ Department of Materials Science, Darmstadt University of Technology \\ Petersenstr. 23, 64287 Darmstadt, Germany
}

\begin{abstract}
Grazing-incidence X-ray diffraction supplemented with atomic force microscopy and secondary ion mass spectroscopy were applied to the characterization of films deposited by laser ablation on cold substrates from $\mathrm{YBaCuO}$ targets and subsequently irradiated with additional laser pulses of lower energy density. Evolution of X-ray diffraction pattern was observed as a function of irradiation dose. For the as-deposited films the pattern was typical of the amorphized solids. For the films irradiated with doses higher than the threshold, the pattern was enriched with the diffraction peaks, whose general features, like peak positions, widths and relative intensities were almost independent of the dose. The size of the crystallites was deduced from the peak widths to be not smaller than 12-16 nm. Comparison of the pattern with patterns of known phases indicates that, apart of the amorphous component, a structure with an admixture of some new metastable or high temperature phase(s) is formed during the process of pulsed laser annealing. The atomic force microscopy observations revealed that the surface roughness shows a pronounced minimum at low irradiation doses. The secondary ion mass spectroscopy investigation confirms that the strongest chemical changes (increase in concentration of yttrium and copper) due to irradiation with higher doses are observed in the near-surface film material.
\end{abstract}

PACS numbers: 52.38.Mf, 81.15.-z, 68.55.-a 


\section{Introduction}

Pulsed laser deposition (PLD) is one of widely used methods of obtaining thin oxide films. It is a complicated process carried out in a low-pressure oxygen atmosphere. The pulse energy from a high power excimer laser is absorbed on the target surface resulting in its rapid heating. As a consequence, an explosive generation of thermal stresses occurs, and near-surface layers of the target are melted and evaporated. A characteristic plasma plume is formed from the ejected material. The plume is condensed subsequently on the substrate surface, where finally a solid layer is formed. Its structural properties depend on the substrate temperature, the ambient oxygen pressure, geometry of deposition and other parameters of the process.

Film surfaces produced by this method exhibit high roughness, and complex morphology [1-3]. Understanding of the physical processes involved in the film formation is important to improve growth of layers with desired properties. In studies of the $\mathrm{Y}-\mathrm{Ba}-\mathrm{Cu}-\mathrm{O}$ system, most attention was usually paid to the structure of superconducting $\mathrm{YBCO}\left(\mathrm{YBa}_{2} \mathrm{Cu}_{3} \mathrm{O}_{7-\delta}\right)$ phase. Some other nonsuperconducting phases of the system show also interesting properties due, e.g., to strong pyroelectric effect, along with a high temperature coefficient of resistivity [4, 5]. Preparation of crystalline layers requires maintaining of the substrate at a high temperature during or after deposition. The fully crystallized layers are usually obtained in the PLD process due to annealing if substrate is heated to a high temperature during deposition. A post-deposition laser irradiation ("laser annealing" or "photodoping") is another method of modification of the film structure and its properties [6, 7]. The post-deposition laser treatment of solids finds many practical applications and for this reason is a subject to intense research activity. It was shown that application of excimer laser can significantly lower the crystallization temperature of some amorphous compounds [7]. In superconducting YBCO films, the laser irradiation was found to induce persistent and metastable insulator-metal and insulator-superconductor transitions [8]. Generally, the laser treatment is found to facilitate structural transformations of the irradiated medium [9]. The exact nature of the involved interactions is not fully understood. A crucial role in initializing the laser induced structural changes is most probably played by the near-surface material of the film $[9,10]$. These facts motivated us to study the possible aspects of the complicated structural changes induced by laser treatment in initially amorphous material. In this work, we focused on the study of structural modifications in the films formed by $\mathrm{PLD}$ from $\mathrm{YBaCuO}$ targets due to post-deposition irradiation by additional laser pulses of various energy densities (fluencies) far below the ablation threshold. 


\section{Experimental}

The films were prepared on glass and two kinds of monocrystalline substrates: Si (100), and $\mathrm{LaAlO}_{3}$ (110). The deposition was carried out in ambient oxygen atmosphere under pressure of 400 mTorr. The stoichiometric target was ablated with $248 \mathrm{~nm} \mathrm{KrF}$ excimer laser of pulse duration $30 \mathrm{~ns}$ and repetition rate $10 \mathrm{~Hz}$ at the energy density (fluence) of $1.5 \mathrm{~J} / \mathrm{cm}^{2}$ per pulse. All samples were deposited on cold substrates ( $T=300 \mathrm{~K}$ ) positioned in "on-axis" geometry by applying 3000 pulses. As a result of the above procedure, the films of thickness about $1 \mu \mathrm{m}$ and of high surface roughness were grown. After preparation, the samples were exposed to additional laser treatment. The same laser, as for film deposition, was used but the pulse energy density was kept in the range of $25-100 \mathrm{~mJ} / \mathrm{cm}^{2}$, much below the ablation threshold. The repetition rate of the annealing pulses was kept at $1 \mathrm{~Hz}$. To obtain a reference X-ray diffraction pattern, the films of superconducting YBCO phase deposited in standard "off-axis" geometry on $\mathrm{SrTiO}_{3}$ and $\mathrm{LaAlO}_{3}$ were studied.

The X-ray diffraction patterns were recorded by a powder diffractometer installed at the B2 beamline at Hasylab (Hamburg), using parallel beam monochromatized with a Ge(111) double crystal monochromator. The diffractometer was equipped with a set of parallel horizontal foils (of angular aperture $0.1^{\circ}$ ) at the diffracted beam. The patterns reported in this work were recorded with monochromator set to $\lambda=1.2065(4) \AA$. The wavelength was determined by a least squares procedure for silicon NBS $640 \mathrm{~b}$ standard $\left(a_{\mathrm{Si}}=5.43094 \AA\right)$. The incident beam geometry with grazing angle fixed at $0.5^{\circ}$ was employed during the measurements; this allowed obtaining optimal X-ray signal/noise ratio, almost free of background originating from the substrate.

Sample surfaces were studied by Digital Instruments atomic force microscope (AFM) and the secondary ion mass spectroscopy (SIMS) measurements were performed with CAMECA IMS $6 \mathrm{~F}$ device using cesium primary ions and positive secondary ions $\mathrm{MCs}^{+}(\mathrm{M}=\mathrm{Y}, \mathrm{Ba}, \mathrm{Cu}, \mathrm{O}, \mathrm{Si})$.

\section{Results and discussion}

In Fig. 1 the diffraction patterns are shown of films deposited on Si substrates and treated with $1,3,5$, and 10 annealing pulses, each of fluency $100 \mathrm{~mJ} / \mathrm{cm}^{2}$ (curves B1, B3, B5, B10, respectively) and, for comparison, of the as-deposited non-irradiated sample (curve B0). The latter pattern is characteristic of amorphous materials. The diffraction peaks due to crystallization induced by laser irradiation of the initially amorphized material appear after first 1-2 annealing pulses. The observed line widths of the most intense reflections in curves B3-B10 are highly similar, indicating that the sizes of the crystallites (estimated to be not smaller than 12-16 $\mathrm{nm}$ from Scherrer formula) practically do not depend on number of 
pulses applied. The same characteristic diffraction pattern was found for the investigated samples treated with irradiation dose (understood in this work as a product of the pulse fluency and the number of annealing pulses) of $240-270 \mathrm{~mJ} / \mathrm{cm}^{2}$ and higher, independently of the kind of substrate.

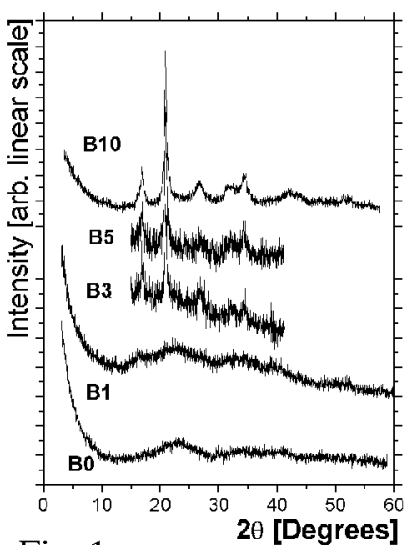

Fig. 1

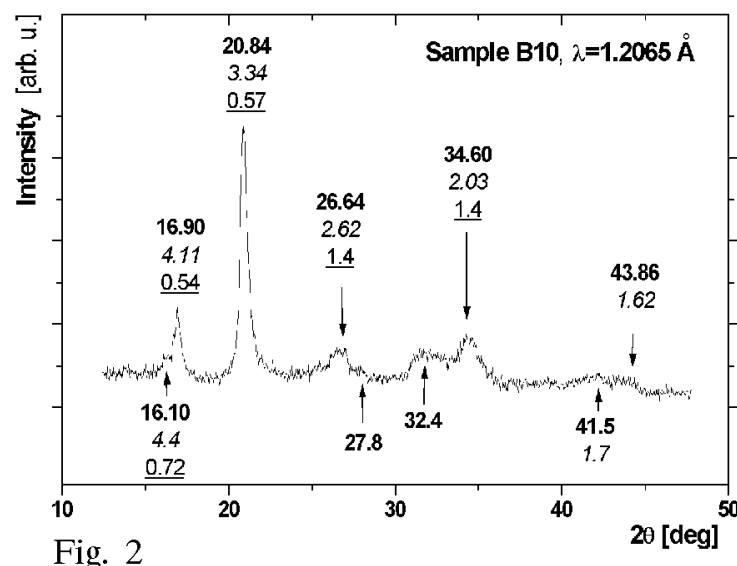

Fig. 2

Fig. 1. X-ray diffraction patterns of $\mathrm{Y}-\mathrm{Ba}-\mathrm{Cu}-\mathrm{O}$ films deposited on cold $\mathrm{Si}(100)$ and annealed with laser pulses applied after deposition. The $n$ in the $B n$ symbol denotes the number of the irradiation pulses. The annealing pulse energy density was $100 \mathrm{~mJ} / \mathrm{cm}^{2}$. Fig. 2. X-ray diffraction pattern of sample B10. Numerical values in bold and underlined refer to fitted peak position and FWHM, respectively. The interplanar spacing, $d$ (in angstroms) is indicated in italics.

The results of analysis of the pattern obtained for sample B10 are shown in Fig. 2. The observed pattern is definitely different than that recorded for the superconducting $\mathrm{YBa}_{2} \mathrm{Cu}_{3} \mathrm{O}_{7-\delta}$ phase [11]. Width and shape of some weak and broad bands at higher $\theta$ angles $\left(\approx 32^{\circ}-35^{\circ}\right.$ and $\left.\approx 41^{\circ}-44^{\circ}\right)$ suggest their multipeak composition. It can be assumed that the observed pattern is probably formed by a multiphase structure containing copper and yttrium oxides, which is supported by the results of SIMS measurements, as discussed later. The phase(s) contributing to the diffractogram are assumed to be metastable or stable at high temperatures only. Extensive efforts of identification were unsuccessful. In particular, none of phases of the $\mathrm{Y}-\mathrm{Ba}-\mathrm{Cu}-\mathrm{O}$ system or any of its subsystems, as compared with the available literature data with the aid of ICDD powder diffraction database, could be ascribed to the pattern (see [12] and references therein).

The film surfaces were studied by the AFM technique. In Fig. 3, the patterns are shown for samples deposited on $\mathrm{LaAlO}_{3}$ substrate. D0 refers to as-deposited sample without additional laser irradiation, D1 and D2 to samples irradiated with 5 pulses of fluency 40.6 and $55.4 \mathrm{~mJ} / \mathrm{cm}^{2}$, respectively (irradiation dose: 0,203 , and $277 \mathrm{~mJ} / \mathrm{cm}^{2}$, respectively). As expected, the surface roughness is high for all investigated samples, reaching for as-deposited films the values of 300-400 nm 
peak-to-peak, typical of the samples grown in on-axis geometry [3]. The variety of morphological features, reported in some papers [3, 4], has not been found in the investigated material. Unexpectedly, we found that the surface of samples annealed with small irradiation doses (of the order $200-250 \mathrm{~mJ} / \mathrm{cm}^{2}$ ) was clearly more smooth, of smaller granularity, than that of as-deposited samples annealed with higher doses (see Fig. 3). This can signify some selective decomposition or evaporation of near-surface material when treated with the low irradiation doses. Further increase in the irradiation dose resulted in a monotonic increase in the size of observed surface features. The appearance of a surface roughness minimum as a function of the irradiation dose was also observed in (not shown here) AFM images of samples deposited on silicon and glass substrates. Surface details of size comparable to the size of crystallites deduced from X-ray line width were not observed.
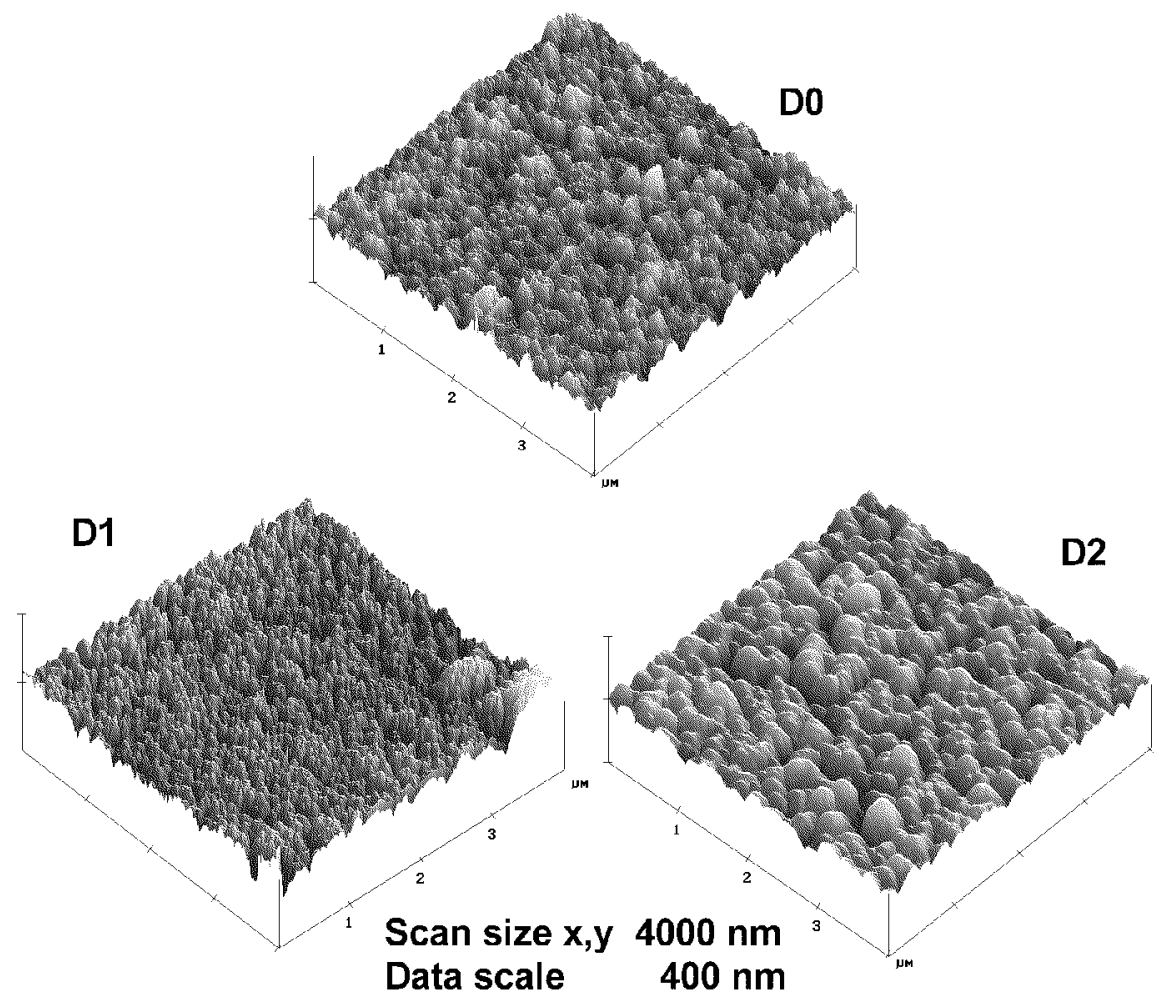

Fig. 3. Variation of layer roughness on laser annealing conditions, illustrated by AFM patterns obtained for layers deposited on $\mathrm{LaAlO}_{3}$ substrate. D0 - sample without additional laser irradiation, D1 and D2 - irradiated with 5 pulses of fluencies 40.6 and $55.4 \mathrm{~mJ} / \mathrm{cm}^{2}$, respectively.

The SIMS technique was applied to study in-depth concentration changes of the elements in the samples due to laser irradiation. The recorded SIMS depth 
profiles of atomic distribution for $\mathrm{Y}, \mathrm{Ba}, \mathrm{Cu}, \mathrm{O}$, and $\mathrm{Si}$ elements in two films deposited on silicon substrates are shown in Fig. 4. Both studied samples were irradiated with the same irradiation dose of $500 \mathrm{~mJ} / \mathrm{cm}^{2}$. Sample A10 was irradiated with 10 laser pulses of fluency $50 \mathrm{~mJ} / \mathrm{cm}^{2}$ each, and the sample B5 with 5 pulses of fluency $100 \mathrm{~mJ} / \mathrm{cm}^{2}$. The obtaining of the depth profiles from the non-annealed reference film deposited on silicon was not possible due to strong static charging of the sample. Similar, but less intense effect, was observed for the sample A10. Therefore, the SIMS signal for this sample is slightly shifted from the starting sputtering time. The observed diminishing of charging with increase in annealing energy indicates for an increase in conductivity of the $\mathrm{Y}-\mathrm{Ba}-\mathrm{Cu}-\mathrm{O}$ film due to laser irradiation. A similar phenomenon has been reported in the literature [9].

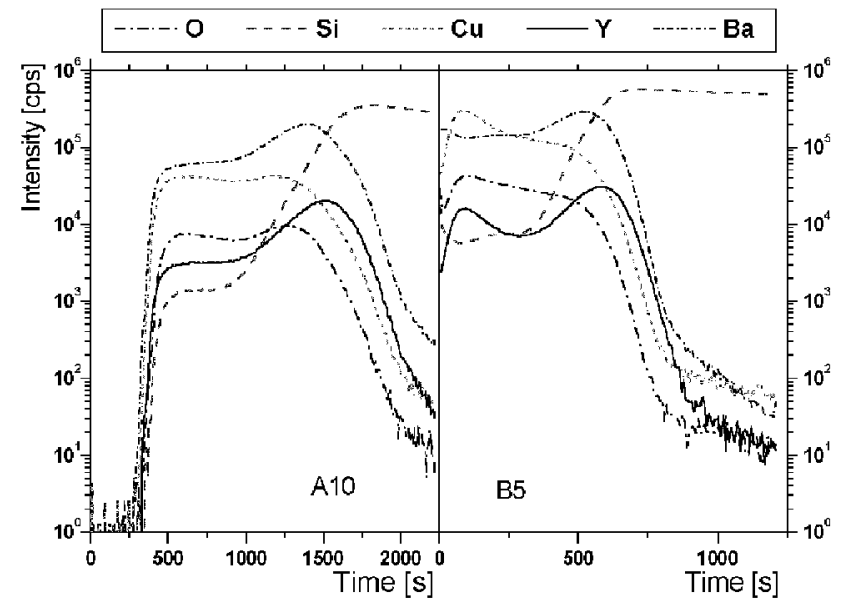

Fig. 4. SIMS depth profiles of atomic concentrations for $\mathrm{Y}, \mathrm{Ba}, \mathrm{Cu}, \mathrm{O}$, and $\mathrm{Si}$ atoms for layers A10 and B5 deposited on Si substrates.

Comparison of results from A10 sample and from stoichiometric YBCO 1-2-3 films characterized by smooth surfaces has shown that the relative intensities of $\mathrm{Y}, \mathrm{Ba}$, and $\mathrm{Cu}$ ion currents are highly similar and can be ascribed to the near-surface part of the layer itself (in the range of about $600-800 \mathrm{~s}$ for $\mathrm{A} 10$ sample). This implies a stoichiometric atomic ratio, $\mathrm{Y}: \mathrm{Ba}: \mathrm{Cu}=1: 2: 3$, in this part of sample. High surface roughness of the samples, confirmed by AFM, results in smearing the signal depth and deteriorates effective in-depth resolution. It influences also the sputtering speed, making especially difficult a precise calibration of the results both with respect to the distance scale and the relative concentrations of the investigated elements (atoms). In particular, it allows only for an approximate localization of film-substrate interface position. Despite these limitations, some general properties of the in-depth distribution of the four elements in the samples can be determined. Concentrations of $\mathrm{Ba}$ and $\mathrm{Y}$ atoms seem to be maximal near the substrate surface, with the maxima slightly shifted with respect to 
each other. This is especially pronounced in sample A10. Irradiation with higher energy density per pulse produced in sample B5 an additional maximum of concentration of the yttrium and copper close to the top surface of the film. Because of insufficient information about the sputtering yield of silicon in the film matrix it is more difficult to evaluate the silicon concentration in the films. The results imply a significant migration of silicon into the layer during deposition, which leads to relative concentration of $\mathrm{Si}$, over the whole film depth, of the order of $10^{-3}-10^{-2}$ $(0.1-1 \%)$. As we obtained similar diffraction patterns in layers deposited on various substrates, the conclusion is that this high silicon admixture does not influence formation of the observed diffraction structure during laser irradiation.

The relative constancy of the diffraction pattern was observed for the films irradiated with total energy densities of about $250-270 \mathrm{~mJ} / \mathrm{cm}^{2}$ up to $1200 \mathrm{~mJ} / \mathrm{cm}^{2}$, the highest applied in the present investigation. Its general features, like line positions, relative intensities, and widths did not depend significantly on the dose. This indicates that crystallites involved in creation of the respective diffraction lines do not grow significantly with the dose. Instead, the observed increasing intensity of the peak pattern implies increase in the number, or the volume fraction of the crystallites with dose. There are many possible mechanisms that can result in such behavior. Although the present results could suggest a stable crystallite size with irradiation, it must be stressed that more information on the structure and properties of films in various annealing schemes and ambient conditions will be necessary to clarify this issue. Attempts to grow the crystalline grains of the structure should also involve the irradiation doses much above the upper limit applied in the present report. More complete analysis of the case and discussion will be published elsewhere.

\section{Conclusions}

The presented work provides preliminary characterization of films deposited by laser ablation on cold substrates from $\mathrm{YBaCuO}$ targets and irradiated with additional laser pulses. The grazing-incidence $\mathrm{X}$-ray diffraction supplemented with AFM and SIMS reveals complicated structural changes in the films. The diffraction patterns range from the pattern typical of amorphized solids observed in as-deposited samples to the relatively stable pattern obtained after irradiation by a dose higher than a certain threshold. The AFM results demonstrate that the surface roughness shows a pronounced minimum for samples irradiated with low doses and the SIMS investigation confirmed that the strongest changes due to irradiation are observed in the near-surface film material.

\section{Acknowledgments}

This work has been supported by the State Committee for Scientific Research (Poland), grants no. 2P 03B 09516 and 2P03B 148 14. The synchrotron 
measurements at HASYLAB (Hamburg) were supported by the TMR-Contract ERBFMGEECT950059 of the European Community.

\section{References}

[1] S. Proyer, E. Stangl, M. Borz, B. Hellebrand, D. Bauerle, Physica C 257, 1 (1996).

[2] M.D. Strikovsky, E.B. Klyuenkov, S.V. Gaponov, Appl. Phys. Lett. 63, 1146 (1995).

[3] T.J. Jackson, S.B. Palmer, J. Phys. D 27, 1581 (1994).

[4] V.D. Okunev, Z.A. Samoilenko, V.M. Svistunov, A. Abal'oshev, E. Dynowska, P. Gierłowski, A. Klimov, S.J. Lewandowski, J. Appl. Phys. 85, 7282 (1999).

[5] D.P. Butler, Z. Celik-Butler, R. Adam, R. Sobolewski, J. Appl. Phys. 85, 1075 (1999)

[6] V.D. Okunev, Z.A. Samoilenko, A. Abal'oshev, P. Giertowski, A. Klimov, S.J. Lewandowski, Appl. Phys. Lett. 75, 1949 (1999).

[7] H. Hiramatsu, Y. Ohki, D.-S. Shin, L.-H. Choi, Y.-T. Kim, Ferroelectric Thin Films VII Symp., Eds. R.E. Jones, R.W. Schwartz, S.R. Summerfelt, I.K. Yoo, Mater. Res. Soc., Warrendale 1999, p. 293.

[8] A.G. Panfilov, A.I. Rykov, S. Tajima, A. Yamanaka, Phys. Rev. B 58, 12459 (1998).

[9] S.J. Lewandowski, G. Jung, V.D. Okunev, Z.A. Samoilenko, V.A. Isaev, A. Abal'oshev, P. Gierłowski, A. Klimov, S. Barbanera, Proc. SPIE 4086, 518 (2000).

[10] H. Kwak, K.C. Chou, J. Guo, H.W.K. Tom, Phys. Rev. Lett. 83, 3745 (1999).

[11] W. Wong-Ng, R.S. Roth, F. Beech, K.L. Davis, Adv. X-ray Anal. 31, 359 (1988).

[12] PDF-3 Database, ICDD, Newtown Square, PA 1999. 